\title{
Die Medizin - ein Werkzeug, das mit Bedacht einzusetzen ist
}

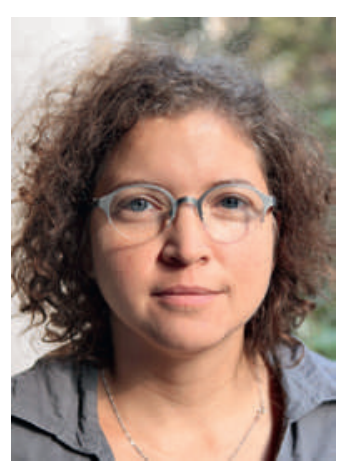

Samia Hurst

\author{
Wer nur einen Hammer hat, \\ für den sieht jedes Problem wie ein Nagel aus. \\ (Abraham Maslow, \\ Die Psychologie der Wissenschaft; 1966)
}

Wenn von «Medikalisierung» die Rede ist, wird vor der Tendenz gewarnt, medizinische Probleme dort zu sehen, wo keine sind. Bei der letzten Überarbeitung des psychiatrischen Diagnosehandbuchs (DSM 5) beispielsweise gab es lebhafte Diskussionen darüber, dass jetzt auch bei Menschen in einer Trauerphase die Diagnose einer schweren Depression gestellt werden kann, was bisher ein Ausschlusskriterium war [1]. Dahinter steht die Absicht, die Erstattung von Behandlungskosten zu ermöglichen, wenn eine Therapie für den Betroffenen sinnvoll erscheint. Es birgt aber natürlich auch die Gefahr, dass ein schmerzhaftes Ereignis, das Teil des Lebens ist, zu einem medizinischen Problem mit einer medizinischen «Lösung» gemacht wird.

\section{«Es fällt schwer, einfach nichts} zu tun.»

2 Marmot M. The Status Syndrome; How Social Standing Affects Our Health and Longevity. London: Bloomsbury Publishings; 2004.

3 WHO. Socioeconomic determinants of health: the solid facts. Genf: Weltgesundheitsorganisation; 2003.

4 Spoerri A, Zwahlen M, Egger M, Gutzwiller F, Minder C, Bopp M. Educational inequalities in life expectancy in German speaking part of Switzerland 1990-1997: Swiss National Cohort. Swiss Med Wkly. 2006;136(9/10):145-8.

\footnotetext{
* Prof. Dr. Samia Hurst ist Professorin am Institut Ethique, Histoire, Humanités der Medizinischen Fakultät der Universität Genf
}

samia.hurst[at]saez.ch seine Mutter verliert. Wir vertrauen der Medizin, und wir fordern immer mehr von ihr. Manchmal vielleicht zu viel. In jedem Fall sind die Mechanismen der Medikalisierung hartnäckig und stossen auf wenig Widerstand.

Medikalisierung beruht in der Regel auf Fehlern. Wenn man sich darauf einlässt, Probleme zu behandeln, die gar nicht medizinischer Natur sind, ist das Scheitern vorprogrammiert. Das hat Nebenwirkungen: Man kann sich gemeinsam einbilden, etwas gegen das Problem zu tun, obwohl dies nicht der Fall ist. Am Ende eines Lebens Therapien ohne echte Wirkung weiterzuführen, nur um dem Patienten zu zeigen, dass man ihn nicht im Stich lässt, oder berufsbedingten Burnout nur mit den Mitteln der Psychiatrie zu behandeln, ohne auch die soziale Komponente zu berücksichtigen - all das ist sicherlich verlockend, droht aber zu einer reinen Alibiübung zu verkommen. Wie jedes einzelne ihrer Instrumente ist auch die Medizin als Ganzes ein Werkzeug, das mit Bedacht anzuwenden ist - nur dort, wo es auch geeignet ist.

Aber wofür ist die Medizin geeignet? Da sind wir bei der entscheidenden Frage.

Das Bestreben, nur ja nicht in die Medikalisierung sozialer Probleme zu verfallen, kann auch dazu verleiten, grundsätzlich um alles in der Medizin einen Bogen machen zu wollen, was auch nur entfernt «politisch» ist. Wir müssen uns aber bewusst sein, dass es auch Situationen gibt, in denen es legitim ist, das Instrumentarium der Medizin einzusetzen, auch wenn es sich eigentlich um ein gesellschaftliches Problem handelt. Die Auswirkungen unserer Gemeinschaftsstrukturen auf die Gesundheit beispielsweise werden zunehmend auch wissenschaftlich untersucht, auf mehreren Kontinenten und auch in der Schweiz [2-4]. Mangelnde Kontrolle über die eigene Arbeit und die eigenen Lebensumstände schlagen sich auf Morbidität und Lebenserwartung nieder. Dieser Effekt ist nun aus dem Bereich der politischen Meinung in das Reich der Tatsachen überführt worden. Man kann darüber streiten, wie damit umzugehen ist, wie er in unsere kollektiven politischen Entscheidungen einfliessen soll, aber leugnen kann man ihn nicht. Also müssen wir auch in der Lage sein zu erkennen, wann unsere Werkzeuge am Platz sind: beobachten, diagnostizieren, hinweisen, auch wenn man keine Behandlungslösung hat - dies sind Aufgaben, die Sache der Medizin sein können. 